\title{
Development of Double-arm Rock Drilling Car for Full Face Tunnelling
}

\author{
Hongli Wang ${ }^{1, ~ *}$, Wei Bao ${ }^{1}$, Xiantang Zhang ${ }^{1}$, Shuyue Wang ${ }^{2}$, Wenquan Cheng ${ }^{1}$, Lixin Song ${ }^{3}$ \\ ${ }^{1}$ Academic Affairs Department of Tai'an Campus, Shandong University of Science and Technology, Tai'an, China \\ ${ }^{2}$ Senior High School, Shandong Tai'an Great Wall Middle School, Tai'an, China \\ ${ }^{3}$ Technology Department, Qingdao Dabang Rock Drill Company Ltd., Qingdao, China
}

Email address:

skdwhl@163.com (Hongli Wang),86057618@163.com (Wei Bao),zzxhtm@163.com (Xiantang Zhang), tawsy2001@163.com (Shuyue Wang),syk201@163.com (Wenquan Cheng),dbzj-170@163.com (Lixin Song)

${ }^{*}$ Corresponding author

\section{To cite this article:}

Hongli Wang, Wei Bao, Xiantang Zhang, Shuyue Wang, Wenquan Cheng, Lixin Song. Development of Double-arm Rock Drilling Car for Full Face Tunnelling. Journal of Civil, Construction and Environmental Engineering. Vol. 4, No. 4, 2019, pp. 81-86.

doi: $10.11648 /$ j.jccee.20190404.12

Received: August 29, 2019; Accepted: September 16, 2019; Published: September 30, 2019

\begin{abstract}
Blasting excavation is widely used in coal mine roadway excavation, its construction method is flexible, convenient, low cost and adaptable. It can excavate mine tunnels of any shape and length under any environmental conditions. However, the main rock drilling equipment used in the mine is pneumatic rock drilling equipment generally. Although these equipment are simple and reliable, their drilling efficiency is low, the degree of automation is poor, and the working conditions are bad, especially the ability to drill deep holes and large holes is poor, which can not meet the needs of large structural parameters mining method. The newly developed double-arm rock drilling rig for full-face roadway excavation is suitable for the current common construction method of roadway blasting driving. It can replace the traditional pneumatic rock drilling equipment, and drill large holes and small holes simultaneously. It fully meets the requirements of blasting technology. It can not only drill and excavate holes, but also drill roof, side wall and floor bolts holes, to achieve high and low position of drilling operations, for improving the efficiency of roadway excavation, promoting the level of coal mine machinery and equipment in China, these have important practical significance.
\end{abstract}

Keywords: Full Section, Excavation, Drilling Rock, Drilling Car

\section{Introduction}

Blasting excavation is widely used in coal mine roadway excavation. The construction method is flexible, convenient, low cost and adaptable. It can excavate mine tunnels of any shape and length under any environmental conditions. But the main rock drilling equipment used in the mine is pneumatic rock drilling equipment. Although these equipment are simple and reliable, they have low drilling efficiency, poor automation and poor working conditions, especially the poor ability to drill deep holes and large holes is poor, which can not meet the needs of large structural parameters mining method. A new type of double-arm rock drilling rig for full section driving is developed, which is suitable for the current common blasting construction mode and can replace the traditional pneumatic rock drilling equipment. It has important practical significance for improving the efficiency of roadway excavation and promoting the improvement of the level of coal mine machinery equipment in our country.

\section{Development Ideas}

In order to effectively solve the problems: under mine cutting is difficult, blasting breakstone is large and not easy to throw out, it is difficult to bore holes near the wall and to form smooth blasting, etc. To ensure the construction of cut hole and hollow hole, and prevent the cut eye, the center eye and the hollow eye from penetrating each other. By studying the drilling mechanism of drilling rig and the cutting blasting technology of medium-deep holes, and scientifically utilizing the technology of enlarging the blasting free surface with 
hollow holes in the middle, a new double-arm rock drilling rig for full section driving has been developed (see with: figure 1). This drilling rig is a double-arm caterpillar walking mechanism, which can drill both large holes and small holes at the same time, its fully meets the requirements of blasting technology. It mainly includes: small chassis crawler, matching down-the-hole driller and hydraulic fast rock driller, as well as hydraulic system, operating system and so on. [1]

The down-the-hole driller can drill the central cutting hole with a diameter of $120 \mathrm{~mm}$ and a depth of $3 \mathrm{~m}$. Hydraulic fast rock driller is a new type of drill developed independently, its has independent impact, independent rotary cutting and impact-rotary functions. It can rotate 360 degrees at random, and drill holes with a diameter of $38 \mathrm{~mm}$ at any position on the working face, and it can drill holes near the wall to the greatest extent, compensation positioning is accurate, and which ensure the forming effect of the roadway. It not only can drill rock holes, but also can drill bolt holes of roof, side wall and floor, which achieves drilling operations at different positions of high and low, and improves the speed of roadway excavation and supporting. [2]

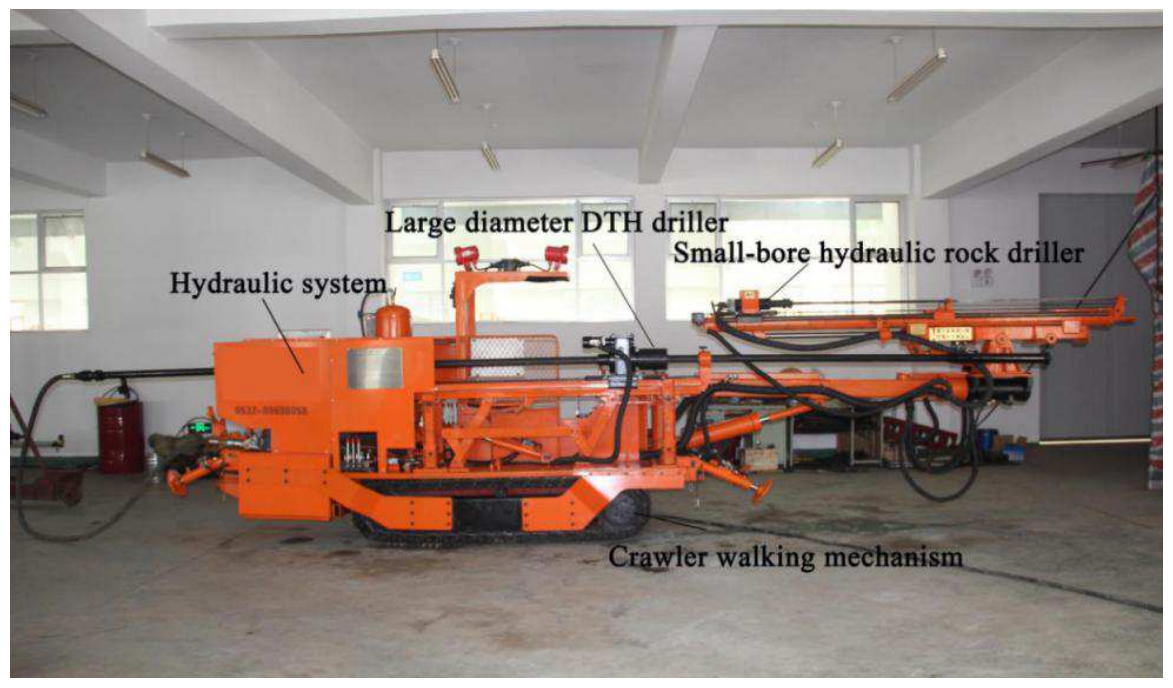

(The main structure: Large diameter DTH driller, Small-bore hydraulic rock driller, Hydraulic system, Crawler driving mechanism.)

Figure 1. Product drawing of double-arm rock drilling rig for full face tunneling.

\section{Key Technologies}

(1) It is necessary to optimize the chassis structure design, and to design the special structure of driving wheel, supporting wheel and guiding wheel, so that they can have better support body and flexibility under different conditions. [3]

(2) The down-the-hole driller of drilling car can mechanically form a larger free surface for drilling holes. In addition, the hydraulic drilling rigger can freely adjust the drilling angle, drill small-diameter cutting holes, auxiliary holes and peripheral boreholes, and can drill holes near the wall to the greatest extent, so as to solve the problems of large number of straight-hole cutting holes, large charge, high parallelism requirement of boreholes, difficult cutting, low utilization rate of borehole, and other problems.

(3) Blasting technology and method optimization. Two large central holes formed by mechanical drilling make it easy to explode a large groove one meter in diameter, and save the surrounding cutting holes. The other two large hole pre-embedded charge can exceed the circular footage, which can make the follow-up blasting better, realize bottomless caving and improve the utilization rate of the blasting hole.

(4) By using the equipment of drilling car, the surrounding holes of smooth blasting can be drilled to meet the standard of "accuracy, flat, straight and neat", so as to ensure the forming standard of the roadway, the surrounding rock is not destroyed, the safety of the roadway is ensured, and the supporting materials can be saved.

(5) The production design of the drilling car is based on the principle of blasting. In order to realize that the drilling rig can drill not only large diameter boreholes, but also small diameter boreholes, and realize the rapid mechanized operation of straight-hole cutting blasting, the design idea is to let two rock drills work at the same time.

(6) The drilling car adopts full hydraulic transmission, three-dimensional and omni-directional drilling, which can achieve no dead angle. At the same time, it has the advantages of compact structure, large driving force, wide working range, advanced hydraulic system, simple operation, reliable performance, strong climbing ability and convenient maintenance.

(7) The hydraulic system of the drilling car is water-cooled, and the water discharge powder can be adjusted and controlled without dust. Besides, the drilling rig is equipped with a seat, which makes the driver operate comfortably, conveniently and safely. 


\section{Parameter Calculation}

After preliminary determination of the parameters of the whole crawler machine, the following calculations are needed to estimate whether the basic performance of the crawler machine meets the design requirements and whether the selection of the parameters of the whole machine is reasonable. This is mainly about the calculation of traction performance.

\subsection{Traction Performance Calculation}

Generally, the working conditions used in calculation are: self-weight in the state of using weight, equal speed running with traction load and full throttle on the stubble ground of horizontal section. [4]

(1) Driving Force of Tracked Machinery $P_{q}$

$$
P_{q}=\frac{M_{e} i_{\Sigma} \eta_{c}}{r_{d q}}(k g f)
$$

Where: $M_{e}$--engine Torque, $\mathrm{kgf}$

$i_{\Sigma}$--total transmission ratio of each gear

$\eta_{c}$--total transmission efficiency of each gear

$r_{d q}$--driving wheel power radius, $m$

$\eta_{q}$--radius efficiency of track drive section generally take, $\eta_{q}=0.95$

$$
\begin{gathered}
G_{\text {smas }}=2 L_{o} b q_{p} \\
G_{s \text { max }}=1.5 P_{T N} \\
P_{T N}=(1.1-1.2) P_{T}
\end{gathered}
$$

Where: $G_{s \max }$--maximum usage weight

$L_{o}$--crawler grounding length

$b$--crawler board width

$q_{p}$--generally, $0.35 \sim 0.5 \mathrm{kgf} / \mathrm{cm}^{2}$

$P_{T N}$--rated force

$P_{T}$--traction force

(2) Moving Resistance of Tracked Machinery $P_{f}$

$$
P_{f}=f G_{s} \quad(k g f)
$$

Where: $G_{s}$--usage weight, $k g f$

$f$--resistance coefficient, crawler type generally, 0.1

The results obtained by calculation. $P_{f}=3.45 K N$

According to the resistance of movement in (2) $P_{f}$, The results obtained by calculation $P_{q}$ :

$$
P_{q}=24.45 K N
$$

(3) Travelling velocity $v$

Theoretical velocity

$$
v_{l}=0.377 \frac{n_{e} r_{d q}}{i_{\Sigma}}(\mathrm{km} / \mathrm{h})
$$

$$
v=v=v_{l}(1-\delta) \quad(k m / h)
$$

Where: $n_{e}$--engine speed

$r_{d q}$--driving wheel power radius

$i_{\Sigma}$--driving wheel slip rate (crawler type, generally, 0.07)

The results obtained by calculation: $v=(2.5 \sim 5) \mathrm{km} / \mathrm{h}$

(4) Tracking efficiency of Caterpillar machinery $\eta_{T}$

$$
\eta_{T}=\eta_{c} \eta_{f} \eta_{\delta} \eta_{q}
$$

Where: $\eta_{c}$--total transmission efficiency of each gear

$\eta_{f}$--rolling efficiency

$\eta_{\delta}$--slip efficiency

$\eta_{q}$--track drive belt efficiency (generally, 0.95 )

The results obtained by calculation: $\eta_{T}=0.65$

(5) Adhesion Force of Track Machinery $P_{\psi \delta}$ (Requirements: The adhesion force shall be greater than or equal to the traction force of the crawler mechanism, and greater than or equal to the sum of the resistance.)

$$
P_{\psi \delta}=\psi_{\delta} G_{\psi}
$$

Where: $\psi_{\delta}$--generally, 0.75

$G_{\psi}$--Computational fetching, $3000 \mathrm{~kg}$

The results obtained by calculation:

$P_{\psi \delta}=25.875 K N \quad$ (Compliance with requirements)

\subsection{Percussion Drilling Fragmentation}

When the impact piston impacts the drill tail each time, the stress wave will be formed in the brazing tool, propagate to the rock bottom, and break the rock. According to the wave theory and the rigid piston model, the calculation is carried out step by step. [5]

(1) Piston impact velocity

$$
V_{P}=\sqrt{2 E / M}(\mathrm{~m} / \mathrm{s})
$$

Where: $V_{P}$--piston impact velocity, $\mathrm{m} / \mathrm{s}$

$$
E \text {-- impact energy, } J
$$

$M$--piston mass, $\mathrm{kg}$

(2) Rock penetration coefficient

The force-depth characteristics of rocks can generally be described by a linear relationship:

$$
F=K U
$$

Where: $F$ - -penetration force

$U$-- depth

$K$-- penetration coefficient

Rock penetration coefficient $K$, i.e. the force required to penetrate drill rock unit depth, it is the most important mechanical characteristic to control the penetration drill process. It is closely related to rock firmness, and increases with the increase of bit blade length during penetration. According to the experimental results, it can be expressed as follows:

Actual velocity 


$$
K=6.313 \sqrt{f} \cdot D_{b} \quad(\mathrm{KN} / \mathrm{mm})
$$

Where: $D_{b}$ - the diameter of the bit ( $\mathrm{cm}$, blade length)

\subsection{Cutting Blasting Technology of Medium-Deep Hole}

The cutting effect is the key to the success of roadway blasting. Cutting hole should not only fully break the rock of full depth, but also throw the broken rock fragments out of the slot cavity, to create a good free surface for auxiliary eye and caving eye.

Providing a good compensation space to create a good free surface for the cutting hole, and a reasonable delay time for the slag blasting hole, which are the key factors affecting the cutting blasting effect. By using this equipment technology, slag cut blasting of full-straight-eye can be used, which not only overcomes the shortcomings of drilling slanting hole limited by roadway section and difficult to operate, but also overcomes the shortcomings of straight-hole cutting poor effect. [6]

(1) The application of this equipment creates a good condition for cutting hole blasting to provide enough compensation space and good free surface. Two holes with a diameter of $120 \mathrm{~mm}$ and a certain depth are drilled at the lower central position of the roadway. Compared with the original holes with the same diameter as the cut hole, it can provide more compensation space, meet the requirements of rock fragmentation of the cut hole, and provide more favorable free surface for the cut hole blasting.

(2) The depth of hollow hole is at least $300 \mathrm{~mm}$ deeper than that of cut hole, which provides space for filling slag-throwing cartridge. At the same time, with the birth of the electronic digital detonator, the electronic delay element can achieve an accurate delay of less than $15 \mathrm{~ms}$, which eliminates passivation effect, fully ensure the reliable initiation of the slag-throwing charge, form a good slag-throwing effect, and create conditions for forming a good groove chamber. At the same time, only one detonator is used for cutting blasting, which creates conditions for delaying time interval of $50 \mathrm{~mm}$ between caving hole and surrounding hole, and realizes medium and deep hole blasting in tunneling.

(3) Because the large diameter hollow hole has enough compensation space, the depth of cut hole does not need to be further deepened. It is easy to operate with the same depth as other holes, and the number of cut holes can be reduced appropriately. The formation of a good groove can effectively reduce the number of caving holes and explosive consumption, thus greatly reducing the total length of each cycle, explosive and detonator consumption, improving the driving speed and reducing the cost.

It is concluded that this drilling equipment can effectively improve the blasting effect of tunneling blasting, increase the utilization ratio of blasting holes per cycle footage (up to $90 \%$ ), reduce the number of blasting holes per cycle (down 5-10 holes), reduce the consumption of detonators and the unit consumption of explosives (explosive consumption can be reduced by $0.2 \mathrm{~kg} / \mathrm{m}^{3}$ ). To realize medium and deep hole blasting in roadway excavation, greatly improve the excavation speed.

\section{Technical Parameters}

The main technical parameters of double-arm rock drilling car for full face tunnelling are shown in Table 1 .

Table 1. Main technical parameters of drilling car.

\begin{tabular}{llll}
\hline Name & Item & Unit & Numerical value \\
\hline & Shape size (length * width & $\mathrm{mm}$ & $7450 \times 1100 \times 2385$ \\
& $*$ height) & & \\
& Crawler climbing ability & $\circ$ & 18 \\
& Walking speed & $\mathrm{km} / \mathrm{h}$ & 2.4 \\
& Power supply voltage & $\mathrm{V}$ & $380 / 660$ \\
Complete & Power & $\mathrm{kW}$ & 30 \\
machine & Number of drilling arms & $\mathrm{number}$ & 2 \\
& Adapted hardness & $\mathrm{f}$ & $6 \sim 14$ \\
& coefficient of rock & $\mathrm{kg}$ & 4860 \\
& Total weight & $\mathrm{m}^{3} / \mathrm{min}$ & $6 \sim 9$ \\
\hline
\end{tabular}

\section{Development of Major Institutions}

\subsection{Crawler Chassis Mechanism}

The crawler chassis ((see with: figure 2) is mainly composed of crawler, walking motor, driving wheel, tension cylinder, tension wheel set and other parts. The walking motor drives the caterpillar forward and backward through the reducer driving sprocket. [7]

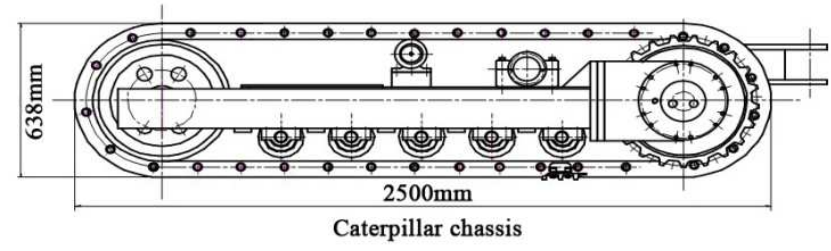

Figure 2. Caterpillar chassis mechanism.

The crawler tensioning mechanism consists of tension wheel group and tension cylinder. Its tightening is driven by tightening the hydraulic cylinder to adjust the tightening wheel. Tightening cylinder is a single-acting form. The tensioning cylinder is installed in the middle of the caterpillar track, which is convenient, practical and beautiful in appearance.

The walking gear reducer is connected with the crawler rack by the anti-loosening high strength bolt. The crawler is fixed with the frame through a shear key and then fastened with high strength bolts. Left and right crawler walking are two completely independent mechanisms.

\subsection{Large Diameter DTH Drill}

The down-the-hole drilling rig is responsible for solving the 
problem of drilling large central hole with a diameter of not less than $120 \mathrm{~mm}$ and a depth of about $3 \mathrm{~m}$. It consists of lifting mechanism, rotator, impactor and propulsion mechanism (see with: figure 3).

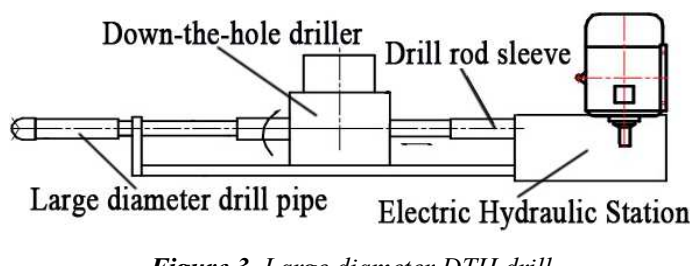

Figure 3. Large diameter DTH drill.

(The main structure: Down-the-hole driller, Drill rod sleeve, Large diameter drill pipe, Electric hydraulic station.)

The lifting mechanism is composed of two hinged cylinders and a support. The lifting cylinder controlling guide rail can be lifted in the support. Drilling operation in different positions is realized. $[8,9]$

The rotater is rotated by a hydraulic motor through a gearbox, and the impactor is installed at the front end of the drill pipe. Under the action of air pressure, its piston continuously impacts the drill bit to realize drilling operation. [10]

Propulsion mechanism is driven chain by hydraulic motor. The chain drives rotator, to realize the drilling rig forward and backward. (see with: table 2)

Table 2. Main technical parameters of DTH drill.

\begin{tabular}{lll}
\hline Item & Unit & Numerical value \\
\hline Borehole diameter & $\mathrm{mm}$ & $90 \sim 130$ \\
Impact Work & $\mathrm{J}$ & 170 \\
Shock frequency & $\mathrm{Hz}$ & 23 \\
Propulsion & $\mathrm{kN}$ & 4 \\
Wind pressure & $\mathrm{MPa}$ & $0.5 \sim 0.7$ \\
Rotational speed & $\mathrm{r} / \mathrm{min}$ & $70 \sim 120$ \\
Torque & $\mathrm{N} \cdot \mathrm{m}$ & $150 \sim 200$ \\
\hline
\end{tabular}

\subsection{Small Hydraulic Rock Driller of Fast and High Efficiency}

The fast and efficient hydraulic rock driller mainly includes manipulator arm, track, propeller and rock drill. It can drill holes in any position within the cross section of $2 \mathrm{~m} * 2 \mathrm{~m}-4 \mathrm{~m} *$ $5 \mathrm{~m}$ in mine roadway (see with: figure 4 ). [11, 12]

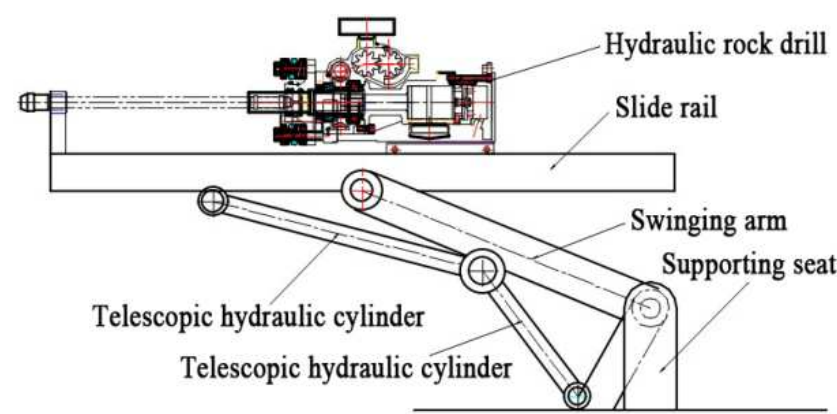

Figure 4. Small hydraulic rock driller of fast and high efficiency.

(The main structure: Hydraulic Rock Driller, Slide rail, Swinging arm, Supporting seat, Telescopic hydraulic cylinder.)
Manipulator arm can cover drilling holes in all-round way, that is to say, drilling rig can achieve multi-directional and multi-angle drilling in front, left and right, up and down. The lifting cylinder of drilling arm is articulated between the drilling arm seat and the drilling arm. The reciprocating motion of the piston rod in its cylinder controls the lifting and landing of the supporting drilling arm. In order to ensure the stability of the drilling arm when it is lifted, a one-way hydraulic lock is installed at the oil inlet. A mechanical arm is embedded in the middle of the drill arm. and an oil cylinder is installed at the other end to control the advance and retreat of the arm. The length of the arm can be adjusted to realize drilling operation in different sections. [13]

The propeller is driven by a hydraulic motor through a gearbox, which makes the driller reciprocate along the guide rail.

The hydraulic rock drill is fixed on the support plate of the rock drill. The rock drill, together with its support plate, moves reciprocally on the guide rail of the propeller under the impetus of the propulsion motor and chain mechanism to complete drilling forward and withdrawing rod backward. In order to adapt to various drilling conditions, throttle speed regulating valve and progressive drilling valve are installed on the hydraulic propulsion system, and drilling speed can be adjusted according to different rocks.

The compensating system is driven the drilling rig by the compensating cylinder along the guide rail, and the front end of the guide rail of the rock drill has a top brace. In rock drilling, the top brace is always on the face of the palm, so as not to move the vacancy and break down when drilling (see with: table 3). [14, 15]

Table 3. Main technical parameters of small hydraulic rock driller.

\begin{tabular}{llll}
\hline Name & Item & Unit & Numerical value \\
\hline & Impact Work & $\mathrm{J}$ & $55 \sim 65$ \\
& Shock frequency & $\mathrm{Hz}$ & $55 \sim 60$ \\
& Torque & $\mathrm{N} \cdot \mathrm{m}$ & $60 \sim 65$ \\
Small & Rotational speed & $\mathrm{r} / \mathrm{min}$ & $250 \sim 300$ \\
Hydraulic & Propulsion & $\mathrm{kN}$ & 2 \\
Rock Driller & Gas-water slag & $\mathrm{kg}$ & $5-8 / 3-5$ \\
& discharge capacity & $\mathrm{MPa}$ & 17 \\
& Oil pressure & $\mathrm{mm}$ & $32 \sim 46$ \\
\hline
\end{tabular}

\section{Conclusions}

The research results have realized the deep integration and innovation of drilling equipment, drilling technology and blasting technology. Compared with foreign full hydraulic drilling trucks, the technical productivity can reach more than $80 \%$, and the characteristics of high reliability, easy maintenance and low price of pneumatic drilling equipment are maintained, which promotes the development of drilling equipment technology and blasting technology in China. This rock drilling car provides advanced technology and methods for the construction of rapid and safe driving in coal mines in China.

(1) Rock drilling rig is a deep combination innovation in 
existing rock drilling technology. It is the first time to optimize the design of DTH drill and rotary percussion drill on crawler chassis, and realize the flexible and efficient operation of the whole machine structure. [16]

(2) Optimizing the key technology of single free surface large hole central hole cutting, using the newly developed $120 \mathrm{~mm}$ large hole down-the-hole drilling machine, the blasting free surface of two $120 \mathrm{~mm}$ intermediate holes is directly realized; at the same time, because the main drill arm is flexible and flexible, the drilling angle can be adjusted freely, and the arm-attached drilling can be maximized, and the standard smooth surface can be drilled. Blasting peripheral holes ensures the standard of roadway forming and the surrounding rock is not destroyed, which ensures the safety of roadway and saves supporting materials.

(3) For the first time, the technology of enlarging the free surface of blasting with hollow holes in the middle is combined with drilling technology, which realizes the optimization of drilling technology and blasting technology, and improves the efficiency of drilling and blasting. The existence of two $120 \mathrm{~mm}$ large holes makes it easy to explode a large groove one meter in diameter and saves 8-10 holes. Two large hole pre-embedded charges and $300 \mathrm{~mm}$ beyond the circular footage can make the subsequent blasting bottomless collapse, reaching $100 \%$ of the utilization rate of the blasting hole, and thoroughly solve the three major problems of difficult cutting, low utilization of the blasting hole and serious destruction of the surrounding rock.

\section{Acknowledgements}

This research was financially supported by Project-oriented National Natural Science Foundation of China (51874187), Shandong Province Science and technology development project (2013GSF12002) and Qingdao West Coast New Area Science and Technology Project.

\section{References}

[1] WANG Hongli, ZHAO Jinqiao, BAO Wei. Study on mechanism of special rotary-impact roof bolter in coal mining [J]. Mining \& Processing Equipment, 2008, 36 (2): 6-10. (in Chinese).

[2] BAO Wei, SUN Wenjin, SONG Hui, etc. Study on key technologies of coal mine rapid drilling equipment and rapid blasting by large diameter straight hole cutting [J]. Metal Mine, 2017 (07): 65-68. (in Chinese).

[3] WANG Hongli, BAO Wei, ZHANG Xiantang, etc. Study on prediction of rotary-impact drilling speed of rock drill [C]. Advances in Engineering Research, ICMEIM, 2017.

[4] WANG Hongli, BAO Wei, XU Taihui, etc. Study on Coal Mine Fast Drilling Equipment and Blasting Key Technology [C]. Advances in Engineering Research, ICEESD, 2017.

[5] ZHAO Tongwu. The research of the energy absorber in a system for measuring the impact energy of rockdrills by means of stress wave [J]. Mining and Metallurgical Engineering, 1987, 7 (2): 1-5. (in Chinese).

[6] LIU Jia. Theoretical and experimental research on static characteristics of mechanism of pneumatic rotary roof bolter [D]. Beijing: Beijing Jiaotong University, 2005. (in Chinese).

[7] ZHAO Xueshe. Present status and development tendency of high efficient roadway driving technology in coal mine [J]. Coal Science and Technology, 2007, (4): 1-4. (in Chinese).

[8] SUN Zhengxin. Discussion on several problems of roof bolter development [J]. Coal Science and Technology, 1999, (12): 37-39. (in Chinese).

[9] ZHAO Tong, LIU Shoukang, TANG Hongping. A study of the efficiency of percussive penetration with joint system [J]. Journal of Rock Mechanics and Engineering, 1994, (1): 19-26.

[10] WU Li, ZHANG Shizhong, LIN Feng. Synthesizing comment on modern rock fragmentation methods [J]. Exploration Engineering, 2000, (2): 49-51. (in Chinese).

[11] ZHAO Tongwu. A study of the efficiency of percussive penetration by wave theory [J]. Acta Metallurgica Sinica, 1980, (3): 263-275. (in Chinese).

[12] DU Changlong. Design and research on impact-rotary roofbolter $[\mathrm{J}]$. Journal of China University of Mining \& Technology, 2002, 31 (1): 92-95. (in Chinese).

[13] Du Changting, Wang Shou, Zhongyi, Wang Bo. Overview of the development of fully hydraulic rock drilling rigs [J]. Pneumatic tools for rock drilling machinery, 2018 (04): 21-26.

[14] Duan Yanhui, Guo Yong, Zhao Hongqiang, etc. Structural improvement of dust collecting cover and internal flow field characteristics of rock drilling rig $[\mathrm{J}]$. Mine machinery, 2016, 44 (05): 13-17.

[15] Qiu Kai. Simulation study of anti-jamming valve of rock drilling rig based on AMESim [J]. Modern manufacturing technology and equipment, 2016 (01): 46-49.

[16] Bao Wei, Song Lixin, Zhao Jinqiao, Bao Dali, Wang Hongli, etc. Development of a fast tunneling compound rock drilling rig [J]. Coal mining machinery, 2015, 36 (08): 209-212. 